\title{
Bedside Ultrasonography-Guided Nasogastric Tube Placement in COVID-19 Patients
}

\author{
Wenhong Gao, MD ${ }^{a, 1}$, Shunji Gao, MD ${ }^{a, 1}$, Qun-An Huang, MD ${ }^{a}$, Huijuan Xiang, MD ${ }^{a, *}$ \\ Department of Ultrasound, General Hospital of Central Military Command Theater, Wuhan 430070, China \\ Received March 31, 2020; revision received April 08, 2020; accepted April 10, 2020
}

\begin{abstract}
Protective measures that are needed when treating patients with highly contagious severe acute respiratory syndrome coronavirus 2 (SARS-CoV-2) add difficulty when performing interventional procedures in isolation wards. We describe two cases of ultrasonography-guided nasogastric tube placement in severe COVID-19 patients, both disabled elderly patients who were unconscious and had complicated underlying diseases. The first is an 83-year-old patient with Alzheimer's, hemiplegia, and epilepsy; the second is a 67-year-old patient with hemiplegia and thoracic deformity. Nasogastric tube indwelling for severe patients like this was essential but challenging. With the help of ultrasonography, intubation proceeded successfully and rapidly. Operator's confidence of appropriate placement was established because of definite tube visualization. These cases demonstrated that bedside ultrasonography in isolation wards can not only facilitate diagnosis but also assist interventional operations.
\end{abstract}

Keywords: Coronavirus; COVID-19; Ultrasonography; Nasogastric Tube

Advanced Ultrasound in Diagnosis and Therapy 2020;02:131-133

DOI: $10.37015 / A U D T .2020 .200024$

$\mathrm{T}$ he global outbreak of Coronavirus Disease 2019 (COVID-19) caused by severe acute respiratory syndrome coronavirus 2 (SARS-CoV-2) has brought new challenges to public health management. Mortality is lower (about 3.9\%) compared to SARS (11\%) and MERS (41\%) [1-3], but SARS-CoV-2 has a basic reproduction number (R0) of at least 1:2.9 in the early stages, making for a high rate of person-toperson transmission $[4,5]$. The high contagiousness of SARS-CoV-2 highlights the need for rigorous prevention management. National Health Commission of the People's Republic of China implemented class-A-level prevention measures for transmission control, although COVID-19 is classified as a class B infectious disease.

According to the hierarchical protection principle for medical practices, medical staff in isolation wards are obliged to wear full set of personal protective equipment, including medical protective suit, protective respirator, goggles, and double layer surgical gloves. Wearing this protective equipment can, however, limit routine physical examinations such as auscultation and percussion. To overcome these limitations, bedside ultrasonography, as a portable and feasible imaging modality, can be used in isolation wards for instant evaluation of cardiac function and detection of hydrothorax and thrombus, among other conditions. Additionally, ultrasonography is also useful in emergent operation guidance. Here, we report two cases of ultrasonography-guided nasogastric tube placement in COVID-19 patients.

\section{Case reports}

\section{Case 1}

An 83-year-old female patient was admitted into isolation wards for positive SARS-CoV-2 nucleic acid detection from a throat swab sample. Before admission, she was receiving treatment for cerebral infarction sequelae in a community hospital. Nasogastric tube indwelling was essential due to cachexia and complicated underlying diseases (hypertension, cerebral infarction, hemiplegia, and senile dementia). Nevertheless, the intubation procedure was impeded not only by

\footnotetext{
${ }^{I}$ These authors contributed equally to this work

* Corresponding author: Department of Ultrasound, General Hospital of Central Military Command Theater, No. 627 Wuluo Road, Wuchang District, Wuhan, Hubei, China

e-mail:xhjyxr@126.com
} unrestricted use, distribution and reproduction in any medium provided that the original work is properly attributed. 
noncooperation but also by age-related thoracocyllosis.

Bedside ultrasonography, performed with a portable ultrasound system (TE7, Mindray biomedical electronic Co., Ltd, Shenzhen, China), was used for tube placement guiding. After attempted insertion of a nasogastric tube for about $15 \mathrm{~cm}$, cervical scanning on the left side was conducted to observe whether the tube was placed correctly in esophagus. When the location was confirmed, the intubation procedure proceeded. When the operation finished, sub-sternum scanning of stomach was performed to further ensure appropriate tip location (Fig. 1).

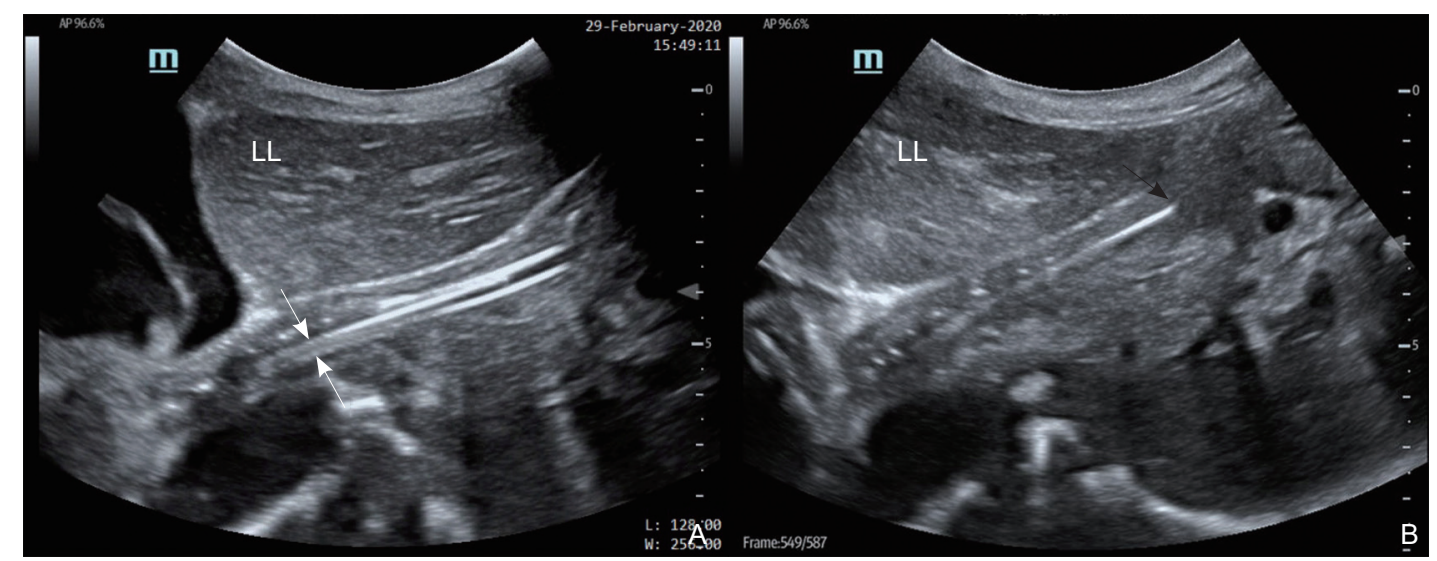

Figure 1 In sub-sternum longitudinal plane, the parallel hyperechoic tube wall (White arrow) can be delineated nicely in esophagus and empty stomach (A), and the tip of the tube (Black arrow) is appropriately placed in the stomach (B). LL, left liver.

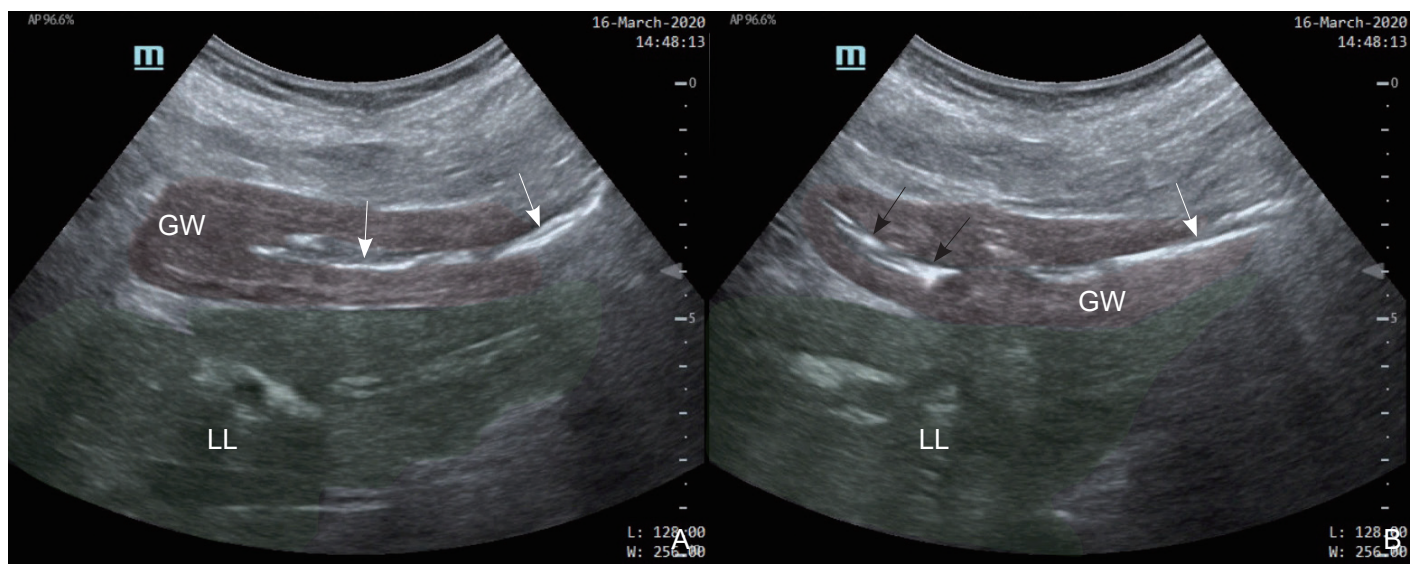

Figure 2 In sub-sternum transversal plane, gastric wall (GW) and gastric mucosa (White arrow) can be observed clearly (A, B), and presence of air bubbles (Black arrow) after tentatively air injection confirmed the appropriated location of nasogastric tube (B). GW, gastric wall; LL, left liver.

\section{Case 2}

A 67-year-old female patient was admitted into isolation wards for fever and bilateral pneumonia. On March 9, she appeared to experience intermittent limb convulsion, without any symptoms of pneumonia. A computed tomography (CT) of the chest showed completely normal pulmonary. Considering her history of epilepsy secondary to cerebral infarction, emergency physician prescribed antiepileptic medicine for symptom control. Five days later, on March 14, this patient developed a fever that reached $38{ }^{\circ} \mathrm{C}\left(100.4{ }^{\circ} \mathrm{F}\right)$, and a new CT scan showed bilateral pulmonary consolidation and pleural effusion. In accordance with the Chinese Clinical Guidance for COVID-19 Pneumonia Diagnosis and Treatment (7th edition) [6], she was diagnosed as having suspected severe pneumonia. Similar to the prior patient, this patient also had anepia and hemiplegia caused by multiple episodes of cerebral infarction. Longterm bed rest and bone degeneration resulted in crooklike thoracic deformity, which made the placement of nasogastric tube quite difficult.

Bedside ultrasonography was performed to help guide the operation. Different from the last patient, whose stomach was well-delineated because of cachexia, this patient's stomach cavity and nasogastric tube could not be well observed because of more distorted thorax and constipation-caused gas disturbance. Therefore, a small amount of air was tentatively injected after intubation, 
while a transducer was used for real-time monitoring sub-sternum. A sign of continuous air bubble rolling indicated that the tube was located appropriately (Fig. 2).

\section{Discussion}

Ultrasonography-guided intervention is a feasible and effective technique. Its history can be traced back to the 1970s [7]. With advantages that include real-time visualization, multiplanar capability, portability, and radiation-free, ultrasonic guidance is now widely used in interventional operations including but not limited to tissue biopsy, minimally-invasive operation, and venous catheterization [8].

Ultrasonography guided intubation of the nasogastric tube and nasojejunal tube have also been reported $[9,10]$. Nasogastric tubes are commonly used in patients for gastrointestinal decompression, medicine administration, and enteral nutrition. In our two cases, both patients were elderly, long-term bedridden, had poor basic condition, and were infected with COVID-19, hence early placement of gastric tube was essential. However, complications of cerebrovascular accident (e.g., hemiplegia, aphasia) and degenerative diseases (e.g., senile dementia, thoracic deformity) made placement of the gastric tube particularly challenging. Conventional blind intubation in this case had a higher risk of misplacement into the trachea, which can result in severe consequences such as pneumonia, pneumothorax, empyema and pulmonary haemorrhage [11]. Nasogastric tubes are made primarily from flexible polyurethane and have a great acoustic impedance that is different from that of soft tissue, making the visualization of the gastric tube possible. By means of cervical esophagus scanning, ultrasonography can identify tube location in the early stages and thus instruct operation.

Furthermore, ultrasonography can also confirm tip location of the inserted tube. Studies have demonstrated good diagnostic accuracy of ultrasonography in the confirmation of appropriate tube placement $[12,13]$. Our observation also confirmed the effectiveness of ultrasonography both under favorable and unfavorable imaging conditions. This is especially valuable in isolation wards, where conventional confirmation methods are usually invalid. In particular, attempted gastric fluids aspiration usually yields nothing because of the impaired gastrointestinal function of severe patients. As for the auscultation method, strict protective wearing in infection wards makes it impossible to complete.

In conclusion, based on our limited observation, we believe bedside ultrasonography should play an important role in nasogastric tube placement in isolation wards as well as in auxiliary assessment.

\section{Acknowledgement}

Funding was provided by Joint Research Project with Health Commission of Hubei Province (No. WJ2019H091), and Wuhan Young Medical Talents Project (PI: Shunji Gao).

\section{Conflicts of Interest}

The authors have no conflict of interest to declare.

\section{References}

[1] Cunha CB, Opal SM. Middle East respiratory syndrome (MERS): a new zoonotic viral pneumonia. Virulence 2014; 5: 650-4.

[2] Liu L. The novel coronavirus SARS-CoV-2: high pathogenecity and its prevention and therapy. Basic \& Clinical Medicine. 2020; 40(4): 433-439.

[3] Mu F, Niu D, Mu J, He B, Han W, Fan B, et al. The expression and antigenicity of a truncated spike-nucleocapsid fusion protein of severe acute respiratory syndrome-associated coronavirus. BMC Microbiol 2008; 8: 207.

[4] Chan JF, Yuan S, Kok KH, To KK, Chu H, Yang J, et al. A familial cluster of pneumonia associated with the 2019 novel coronavirus indicating person-to-person transmission: a study of a family cluster. Lancet 2020; 395: 514-523.

[5] Zhao S, Lin Q, Ran J, Musa SS, Yang G, Wang W, et al. Preliminary estimation of the basic reproduction number of novel coronavirus (2019-nCoV) in China, from 2019 to 2020: A data-driven analysis in the early phase of the outbreak. Int J Infect Dis 2020; 92: 214-217.

[6] China National Health Commission. Chinese clinical guidance for covid-19 pneumonia diagnosis and treatment (7th edition), 2020, March 4.

[7] Holm HH, Kristensen JK, Rasmussen SN, Northeved A, Barlebo $H$. Ultrasound as a guide in percutaneous puncture technique. Ultrasonics 1972; 10: 83-6.

[8] Copelan A, Scola D, Roy A, Nghiem HV. The Myriad Advantages of Ultrasonography in Image-Guided Interventions. Ultrasound $Q 2016$; 32: 247-57.

[9] Gok F, Kilicaslan A, Yosunkaya A. Ultrasound-guided nasogastric feeding tube placement in critical care patients. Nutr Clin Pract 2015; 30: 257-60.

[10] Liu Z, Guo J, Ren W, Tang S, Huang Y, Huang L, et al. Evaluation of ultrasound-guided Freka-Trelumina enteral nutrition tube placement in the treatment of acute pancreatitis. BMC Gastroenterol 2020; 20: 21.

[11] Wallace SC, Gardner LA. Misplacements of enteral feeding tubes increase after hospitals switch brands. Am J Nurs 2015; 115: 44-46.

[12] Brun PM, Chenaitia H, Lablanche C, Pradel AL, Deniel C, Bessereau $\mathrm{J}$, et al. 2-point ultrasonography to confirm correct position of the gastric tube in prehospital setting. Military Medicine 2014; 179: 959963.

[13] Dias FSB, Alvares BR, Jales RM, Franco APV, Silva JEFD, Fabene SMS, et al. The Use of Ultrasonography for Verifying Gastric Tube Placement in Newborns. Adv Neonatal Care 2019; 19: 219-225. 